\title{
DETERMINANTS OF ORTHOGONAL POLYNOMIALS
}

\author{
BY SAMUEL KARLIN AND JAMES MC GREGOR
}

Communicated by Ralph Phillips, January 22, 1962

In the study of coincidence problems for birth-and-death processes $[1 ; 2 ; 3]$ the authors encountered systems of orthogonal polynomials in several variables constructed as follows. Let $\psi(x)$ be a distribution function on the semi-axis $[0, \infty)$ with infinitely many points of increase, and with finite moments of all orders. Let $Q_{n}(x), n=0,1,2, \cdots$ be the orthgonal polynomials for the distribution $\psi$, and taking integers $0 \leqq i_{1}<i_{2}<\cdots<i_{n}$ form the determinant

$$
Q\left(\begin{array}{c}
i_{1}, i_{2}, \cdots, i_{n} \\
x_{1}, x_{2}, \cdots, x_{n}
\end{array}\right)=\operatorname{det} Q_{i_{\alpha}}\left(x_{\beta}\right) .
$$

This is a polynomial in the $n$ variables $x_{1}, \cdots, x_{n}$ and the collection of all such determinants $\left(0 \leqq i_{1}<\cdots<i_{n}\right)$ forms an orthogonal system on the simplex $S=\left\{\left(x_{1}, \cdots, x_{n}\right) ; 0 \leqq x_{1}<x_{2}<\cdots<x_{n}\right\}$. In fact if $\int_{0}^{\infty} Q_{i}(x) Q_{j}(x) d \psi(x)=\delta_{i j} / \pi_{j}$, then (see [2])

$$
\text { (2) } \begin{array}{r}
\int \ldots \int Q\left(\begin{array}{c}
i_{1}, \cdots, i_{n} \\
x_{1}, \cdots, x_{n}
\end{array}\right) Q\left(\begin{array}{r}
j_{1}, \cdots, j_{n} \\
x_{1}, \cdots, x_{n}
\end{array}\right) d \psi\left(x_{1}\right) \cdots d \psi\left(x_{n}\right) \\
=\delta_{i_{1} j_{1}} \cdots \delta_{i_{n} j_{n}} /\left(\pi_{j_{1}} \cdots \pi_{j_{n}}\right) .
\end{array}
$$

A few properties of the polynomials (1) were given in [2]. Here we give an account of additional properties and indicate some interesting generalizations.

We assume throughout that the $Q_{n}(x)$ are normalized so $Q_{n}(0)=1$. This normalization is possible since all zeros of $Q_{n}$ lie in the open interval $(0, \infty)$. The recurrence relation then has the form $-x Q_{n}$ $=\mu_{n} Q_{n-1}-\left(\lambda_{n}+\mu_{n}\right) Q_{n}+\lambda_{n} Q_{n+1}$ where $\mu_{0}=0, \mu_{n+1}>0, \lambda_{n}>0$ for $n \geqq 0$. The constants $\pi_{j}$ are

$$
\pi_{0}=1, \pi_{j}=\frac{\lambda_{0} \lambda_{1} \cdots \lambda_{j-1}}{\mu_{1} \mu_{2} \cdots \mu_{j}}, j \geq 1 .
$$

1. Recurrence relations. The determinantal polynomials

$$
Q\left(\begin{array}{l}
i_{1}, \cdots, i_{n} \\
x_{1}, \cdots, x_{n}
\end{array}\right)
$$

of order $n$ satisfy $n$ different recursion formulas which may be derived from the basic recurrence formula $-x Q(x)=A Q(x)$, where 
$A=\left\|a_{i j}\right\|_{i, j=0}^{\infty}$ and $a_{i j}=\lambda_{i}$ if $j=i+1 ;-\left(\lambda_{i}+\mu_{i}\right)$ if $j=i ; \mu_{i}$ if $j=i$; and 0 otherwise $(i=0,1, \cdots)$.

Applying the composition formula of $[2$, formula (10) $]$ to the recursion formula, we obtain

$$
\begin{aligned}
(-1)^{n} x_{1} x_{2} \cdots x_{n} Q & \left(\begin{array}{c}
i_{1}, \cdots, i_{n} \\
x_{1}, \cdots, x_{n}
\end{array}\right) \\
& =\sum_{0 \leq \alpha_{1}<\alpha_{2}<\cdots<\alpha_{n}} A\left(\begin{array}{c}
i_{1}, \cdots, i_{n} \\
\alpha_{1}, \cdots, \alpha_{n}
\end{array}\right) Q\left(\begin{array}{l}
\alpha_{1}, \alpha_{2}, \cdots, \alpha_{n} \\
x_{1}, x_{2}, \cdots, x_{n}
\end{array}\right)
\end{aligned}
$$

where

$$
A\left(\begin{array}{c}
i_{1}, \cdots, i_{n} \\
\alpha_{1}, \cdots, \alpha_{n}
\end{array}\right)
$$

denotes the minor of $A$ formed with the rows $i_{1}, \cdots, i_{n}$ and the columns $\alpha_{1}, \cdots, \alpha_{n}$.

With the aid of (4) and the Laplace expansion of $n$th order determinants, we obtain

$$
\begin{aligned}
& (-1)^{k} E_{k}\left(x_{1}, \cdots, x_{n}\right) Q\left(\begin{array}{l}
i_{1}, \cdots, i_{n} \\
x_{1}, \cdots, x_{n}
\end{array}\right) \\
& =\sum_{1 \leq \beta_{1}<\cdots<\beta_{k} \leq n} \sum_{0 \leq r_{1}<r_{2}<\cdots<r_{k}} \\
& \times A\left(\begin{array}{c}
i_{\beta_{1}}, \cdots, i_{\beta_{k}} \\
r_{1}, \cdots, r_{k}
\end{array}\right) Q\left(\begin{array}{c}
\left(r_{1} \cdots r_{k}\right)\left(i_{\beta_{1}} \cdots i_{\beta_{k}}\right)^{0} \\
x_{1}, \cdots, x_{n}
\end{array}\right) \\
& k=1,2, \cdots, n \text {, }
\end{aligned}
$$

where $E_{k}\left(x_{1}, \cdots, x_{n}\right)$ is the familiar $k$ th order elementary symmetric function of $n$ variables, i.e., $\sum E_{k}\left(x_{1}, \cdots, x_{n}\right) t^{k}=\prod\left(1+t x_{i}\right)$ and the meaning of the right-hand side in (5) is as follows: we select a $k$-tuple $i_{\beta_{1}}, i_{\beta_{2}}, \cdots, i_{\beta_{k}}$ from $\left(i_{1}, i_{2}, \cdots, i_{n}\right)$ and let $\left(i_{\beta_{1}}, \cdots, i_{\beta_{k}}\right)^{\text {c }}$ consist of the remaining $n-k$ indices which together with $\left(i_{\beta_{1}}, i_{\beta_{2}}, \cdots, i_{\beta_{k}}\right)$ comprise $\left(i_{1}, i_{2}, \cdots, i_{n}\right)$. The summation in (5) is extended over all selections $\left(i_{\beta_{1}}, i_{\beta_{2}}, \cdots, i_{\beta_{k}}\right)$ of $\left(i_{1}, i_{2}, \cdots, i_{n}\right)$ and arbitrary $0 \leqq r_{1}$ $<r_{2}<\cdots<r_{k}$. The set of indices $\left(r_{1}, r_{2}, \cdots, r_{k}\right)$ and $\left(i_{\beta_{1}}, i_{\beta_{2}}, \cdots, i_{\beta_{k}}\right)^{c}$ occurring in

$$
Q\left(\begin{array}{c}
\left(r_{1}, r_{2}, \cdots, r_{k}\right)\left(i_{\beta_{1}}, \cdots, i_{\beta_{k}}\right)^{0} \\
x_{1}, \cdots, x_{n}
\end{array}\right)
$$

are always arranged in increasing order.

The sum occurring in (5) is finite since 


$$
A\left(\begin{array}{c}
i_{\beta_{1}}, \cdots, i_{\beta_{k}} \\
r_{1}, \cdots, r_{k}
\end{array}\right)=0
$$

unless $\left|i_{\beta_{i}}-r_{i}\right| \leqq 1(i=1, \cdots, k)$. The special case $k=1$ in (5) reduces to

$$
\begin{aligned}
-\left(x_{1}+x_{2}+\cdots+\right. & \left.x_{n}\right) Q\left(\begin{array}{c}
i_{1}, \cdots, i_{n} \\
x_{1}, \ldots, x_{n}
\end{array}\right) \\
= & \sum_{k=1}^{n}\left\{\mu_{i_{k}} Q\left(\begin{array}{c}
i_{1}, \cdots, i_{k-1}, i_{k}-1, i_{k+1}, \cdots, i_{n} \\
x_{1}, \cdots, x_{n}
\end{array}\right)\right. \\
& -\left(\lambda_{i_{k}}+\mu_{i_{k}}\right) Q\left(\begin{array}{c}
i_{1}, \cdots, i_{n} \\
x_{1}, \cdots, x_{n}
\end{array}\right) \\
& \left.+\lambda_{i_{k}} Q\left(\begin{array}{c}
i_{1}, \cdots, i_{k-1}, i_{k}+1, i_{k+1}, \cdots, i_{n} \\
x_{1}, \cdots, x_{n}
\end{array}\right)\right\} .
\end{aligned}
$$

The case $k=n$ is (4).

An alternative form of the $k$ th recursion relation is

$$
\begin{aligned}
E_{k}\left(A_{1}, A_{2}, \cdots, A_{n}\right) Q\left(\begin{array}{l}
i_{1}, i_{2}, \cdots, i_{n} \\
x_{1}, x_{2}, \cdots, x_{n}
\end{array}\right) \\
\quad=(-1)^{k} E_{k}\left(x_{1}, x_{2}, \cdots, x_{n}\right) Q\left(\begin{array}{c}
i_{1}, i_{2}, \cdots, i_{n} \\
x_{1}, x_{2}, \cdots, x_{n}
\end{array}\right)
\end{aligned}
$$

where

$$
A_{\nu} Q\left(\begin{array}{c}
i_{1}, \cdots, i_{n} \\
x_{1}, \cdots, x_{n}
\end{array}\right)=\sum_{j} a_{i_{\nu} j} Q\left(\begin{array}{c}
i_{1}, \cdots, i_{\nu-1}, j, i_{\nu+1}, \cdots, i_{n} \\
x_{1}, \cdots, x_{n}
\end{array}\right) .
$$

The following uniqueness assertion holds. If $\phi_{i_{1}}, \cdots, i_{n}\left(x_{1}, \cdots, x_{n}\right)$ is a system of functions which satisfy the full set of all $n$ recurrence relations then

$$
\phi_{i_{1}}, \cdots, i_{n}\left(x_{1}, \cdots, x_{n}\right)=f\left(x_{1}, \cdots, x_{n}\right) Q\left(\begin{array}{c}
i_{1}, \cdots, i_{n} \\
x_{1}, \cdots,{ }^{\pi} x_{n}
\end{array}\right)
$$

where $f$ does not depend on $i_{1}, \cdots, i_{n}$.

2. Christoffel-Darboux formula. The identity

$$
\frac{\lambda_{n} \pi_{n} Q\left(\begin{array}{l}
n, n+1 \\
x, y
\end{array}\right)}{\lambda_{0} \pi_{0} Q\left(\begin{array}{l}
0,1 \\
x, y
\end{array}\right)}=\sum_{k=0}^{n} Q_{k}(x) Q_{k}(y) \pi_{k}
$$


is called the Christoffel-Darboux formula. It has the generalization $\left(\prod_{r=1}^{p} \prod_{s=1}^{m} \frac{\pi_{n+r-1} \lambda_{n+r+o-2}}{\pi_{r-1} \lambda_{r+o-2}}\right) \frac{Q\left(\begin{array}{c}n, n+1, \cdots, n+p-1, n+p, n+p+1, \cdots, n+p+m-1 \\ x_{1}, x_{2}, \cdots, x_{p}, y_{1}, y_{2}, \cdots, y_{m}\end{array}\right)}{Q\left(\begin{array}{c}0,1, \cdots, p-1, p, p+1, \cdots, p+m-1 \\ x_{1}, x_{2}, \cdots, x_{p}, y_{1}, y_{2}, \cdots, y_{m}\end{array}\right)}$

$$
\text { 7) } \sum_{0 \leqslant k_{1}<\cdots<k_{p}} \frac{Q\left(\begin{array}{c}
k_{1}, \cdots, k_{p} \\
x_{1}, \cdots, x_{p}
\end{array}\right) Q\left(\begin{array}{c}
k_{1}, \cdots, k_{p}, n+p, n+p+1, \cdots, n+m-1 \\
y_{1}, \cdots, y_{m}
\end{array}\right)}{Q\left(\begin{array}{l}
0, \cdots, p-1 \\
x_{1}, \cdots, x_{p}
\end{array}\right) Q\left(\begin{array}{l}
0, \cdots, m-1 \\
y_{1}, \cdots, y_{m}
\end{array}\right)} \pi_{k_{1}} \cdots \pi_{k_{p}}
$$

valid for $2 \leqq p \leqq m$. This formula and those of the next section can be derived by inductive arguments based on Sylvesters' identity.

3. The Wronskian identity. The polynomials of the second kind

$$
Q_{n}^{(0)}(x)=\int_{0}^{\infty} \frac{Q_{n}(x)-Q_{n}(y)}{x-y} d \psi(y)
$$

satisfy the recurrence relation for $n \geqq 1$, and the Wronskian identity $Q_{n}(x) Q_{n+1}^{(0)}(x)-Q_{n+1}(x) Q_{n}^{(0)}(x)=-1 /\left(\lambda_{n} \pi_{n}\right)$. For $0 \leqq i_{1}<i_{2}<\cdots<i_{r}$ $<i_{r+1}<\cdots<i_{n}$ let $Q\left(\begin{array}{c|c}i_{1}, i_{2}, \cdots, i_{r} & i_{r+1}, i_{r+2}, \cdots, i_{n} \\ x_{1}, x_{2}, \cdots, x_{r} & x_{r+1}, x_{r+2}, \cdots, x_{n}\end{array}\right)$

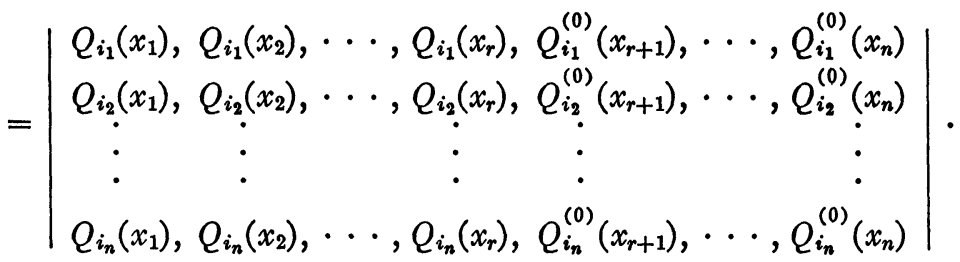

The formulas

$$
\begin{aligned}
& Q\left(\begin{array}{c|c}
n, n+1, \cdots, n+r & n+r+1, n+r+2, \cdots, n+r+k+1 \\
x, y_{1}, \cdots, y_{r} & x, z_{1}, \cdots, z_{k}
\end{array}\right) \\
& =\frac{(-1)^{r+1}\left(\lambda_{0} \pi_{0}\right)^{r+k}}{\pi_{n} \lambda_{n} \lambda_{n+1} \cdots \lambda_{n+r+k}} \prod_{i=1}^{r} Q\left(\begin{array}{ll}
0, & 1 \\
x, y_{i}
\end{array}\right) \prod_{j=1}^{k}\left(\begin{array}{ll}
0, & 1 \\
x, z_{j}
\end{array}\right)
\end{aligned}
$$

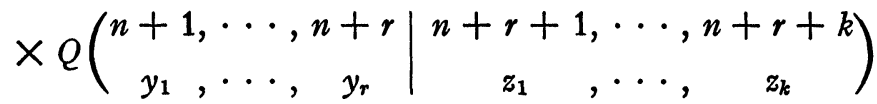

and 


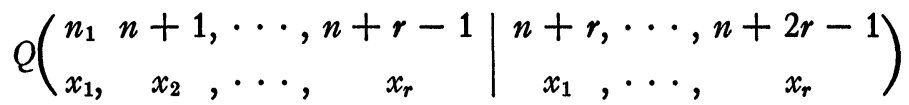

$$
\begin{aligned}
& =\frac{(-1)^{[r(r+1)] / 2}\left(\lambda_{0} \pi_{0}\right)^{r(r-1)} \prod_{1 \leq i<j \leq r}\left[Q\left(\begin{array}{l}
0,1 \\
x_{i}, x_{j}
\end{array}\right)\right]^{2}}{\pi_{n} \pi_{n+1} \cdots \pi_{n+r-1} \lambda_{n} \lambda_{n+1}^{2} \lambda_{n+2}^{3} \cdots \lambda_{n+r-1}^{r} \lambda_{n+r}^{r+1} \lambda_{n+r+1}^{r-1} \cdots \lambda_{n+2 r-2}}
\end{aligned}
$$

are generalizations of the Wronskian identity for the polynomials cited before.

4. Positivity. If $x \leqq 0$ then $Q_{n}(x)>0$. The analogous inequality for the determinants is

$$
(-1)^{n(n-1) / 2} Q\left(\begin{array}{c}
i_{1}, \cdots, i_{n} \\
x_{1}, \cdots, x_{n}
\end{array}\right)>0 \quad \text { if } x_{1}<x_{2}<\cdots<x_{n} \leqq 0,
$$

and this, together with a number of related and sharper inequalities, has been discussed in detail in [2].

5. Continuous analogues. The function $\phi(x, \lambda)=\cos \lambda^{1 / 2} x$ satisfies

$$
\begin{aligned}
\frac{d^{2}}{d x^{2}} \phi & =-\lambda \phi, & 0<x<\infty, \\
\phi(0, \lambda) & =1, \quad \phi_{x}(0, \lambda)=0 . &
\end{aligned}
$$

For fixed $x \geqq 0, \phi$ is an entire function of $\lambda$ and these entire functions are analogous to the polynomials. We form the determinants

$$
\phi\left(\begin{array}{l}
x_{1}, x_{2}, \cdots, x_{n} \\
\lambda_{1}, \lambda_{2}, \cdots, \lambda_{n}
\end{array}\right)=\operatorname{det} \phi\left(x_{i}, \lambda_{j}\right)
$$

where $0 \leqq x_{1}<x_{2}<\cdots<x_{n}$. For each of the above described properties of the determinants (1) there is a similar property of the determinants (10). For example in place of the system of $n$ recurrence relations we have a system of $n$ partial differential equations

$$
(-1)^{k} E_{k}\left(\lambda_{1}, \cdots, \lambda_{n}\right) u=E_{k}\left(\frac{\partial^{2}}{\partial x_{1}^{2}}, \cdots, \frac{\partial^{2}}{\partial x_{n}^{2}}\right) u, k=1,2, \cdots, n,
$$

where $u$ is the determinant (10). The following uniqueness assertion holds. If $u=u\left(x_{1}, \cdots, x_{n} ; \lambda_{1}, \cdots, \lambda_{n}\right)$ has continuous partial derivatives up to order $2 n$ and satisfies all $n$ equations (11) are the closed simplex $0 \leqq x_{1} \leqq x_{2} \leqq \cdots \leqq x_{n}<\infty$, and the boundary conditions 


$$
\begin{aligned}
& u_{x_{1}}\left(0, x_{2}, x_{3}, \cdots, x_{n} ; \lambda_{1}, \cdots, \lambda_{n}\right)=0, \\
& u\left(x_{1}, x_{2}, \cdots, x_{n} ; \lambda_{1}, \cdots, \lambda_{n}\right)=0 \text { if } x_{i}=x_{i+1}, i=1, \cdots, n-1,
\end{aligned}
$$

then

$$
u=f\left(\lambda_{1}, \cdots, \lambda_{n}\right) \phi\left(\begin{array}{c}
x_{1}, \cdots, x_{n} \\
\lambda_{1}, \cdots, \lambda_{n}
\end{array}\right) .
$$

Similar results may be obtained when $\phi(x, \lambda)$ is replaced by the system of solutions of more general Sturm-Liouville problems.

6. Other properties. Many of the results established for the polynomials (1) have analogues dealing with the permanents

$$
Q\left[\begin{array}{c}
i_{1}, \cdots, i_{n} \\
x_{1}, \cdots, x_{n}
\end{array}\right]=\sum_{\sigma} Q_{i_{1}}\left(x_{\sigma(1)}\right) \cdots Q_{i_{n}}\left(x_{\sigma(n)}\right),
$$

the sum running over all permutations $\sigma$ of $(1,2, \cdots, n)$.

Other properties of polynomials in one variable possess analogues for the determinantal systems. We can discuss the properties of zeros, completeness, quadrature formulas, generating functions in the classical cases, etc. Details of these developments, the proofs of the results announced above, and their extensions to eigenfunctions of second order differential operators will be elaborated elsewhere.

\section{REFERENCES}

1. S. Karlin and J. McGregor, Coincidence probabilities, Pacific J. Math. 9 (1959), 1141-1164.

2. - Coincidence properties of birth and death processes, Pacific J. Math. 9 (1959), 1109-1140.

3. - A characterization of birth and death processes, Proc. Nat. Acad. Sci. 45 (1959), 375-379.

Stanford University 